\title{
Universal method for calculation of reliable completion times
}

\section{Универсальный метод вычисления достоверных сроков реализации проекта}

\author{
Yu.B. Kalugin, \\ Military Institute of Rail Transport Troops and \\ Military Communications, St. Petersburg, Russia
}

Key words: civil engineering; construction management; project scheduling; critical path method

\author{
Д-р техн. наук, профессор Ю.Б. Калугин, \\ Военный институт железнодорожных войск \\ и военных сообщений, г. Санкт-Петербург, \\ Россия
}

$\begin{array}{lr}\text { Ключевые слова: } & \text { управление } \\ \text { инвестиционно-строительными проектами; } & \text { пасписание проекта; метод критического пути }\end{array}$

\begin{abstract}
Usually the actual duration of construction projects significantly exceeds the scheduled durations.The reason is the lack of realistic scheduling. This paper presents a universal method for computing the reliable completion time for a construction project with network plans that have imprecise durations. The study is based on the consecutive comparison and calculation of the time distributions for the fulfilment of construction events. The general applicability of the algorithm is demonstrated. The method was used for the calculation of a more realistic time span for the construction of a road. The network schedule is formed on the basis of the flow sheet. A comparison was drawn between the proposed method and traditional techniques. The mean duration of the technological process calculated by a universal method is $30 \%$ more than for a known critical path method. It is confirmed that the traditional method of calculating the time to complete a project is almost always shorter. The method and algorithm presented in this study used Microsoft Excel. The calculations lasted some seconds. Implementation of the universal method will allow for the determination of a more precise duration for the performance of complex works at the planning stage. The suggested methodology can be recommended for use by construction project managers.
\end{abstract}

Аннотация. Обычно фрактическая продолжительность строительных проектов значительно превышает запланированные сроки. Причина - отсутствие инструмента достоверного планирования. В статье представлена универсальная методика для вычисления надежного времени завершения строительных проектов на основе сетевых графиков с неопределенными продолжительностями работ. Исследование основано на последовательном сравнении и вычислении распределений сроков свершений совместных событий графика. Продемонстрирована общая применимость алгоритма. Метод использовался для вычисления достоверных сроков сооружения участка дороги, для чего на основе технологической карты был сформирован соответствующий сетевой график. Сравнение между предложенным подходом и традиционными методами показало, что средняя продолжительность технологического процесса, вычисленного универсальным методом, на 30 \% больше, чем для известного метода критического пути. Подтверждено, что традиционный метод критического пути существенно занижает сроки реализации проекта. Метод и соответствующий алгоритм реализован в cреде Microsoft Excel. Вычисления продлились несколько секунд. Внедрение универсального метода позволит определять достоверные сроки выполнения комплексов работ при планировании проектов. Предложенный подход может быть рекомендован для использования руководителями строительных проектов.

\section{Introduction}

The analysis of the current state of the theory and practice of scheduling illustrates the lack of realistic scheduling.

Therefore, the actual duration of various construction projects significantly exceeds the planned ones [1-6].

Kalugin Yu.B. Universal method for calculation of reliable completion times. Magazine of Civil Engineering. 2016. No. 7. Pp. 70-80. doi: 10.5862/MCE.67.7 
The reason for the significant difference between planned and actual construction durations is, primarily, the impact on the works due to numerous stochastic factors [7]), which can be evaluated using probabilistic estimation [8-12].

The second reason is insufficiently reliable traditional PERT (Program Evaluation and Review Technique) method. The PERT method is generally intended for the calculation of schedules that have certain structures set by unambiguous technological processes. The activity time spans are assumed to follow a general Beta distribution [13-17].

There have been a number of studies $[18,19]$ that consider project scheduling and PERT using fuzzy logic for scheduling under uncertainty. Fuzzy logic has been proposed as an alternative approach for quantifying uncertainty related to project activity duration.

The traditional PERT method uses only the activity time means to calculate the critical path, reducing the stochastic model to a deterministic model. In PERT, three time estimates are required for each activity. The time estimates represent a pessimistic time, an optimistic time, and a most likely time for duration of the activity.

The method assumes that the sum of the mean completion times of activities on the critical path is normally distributed. This allows the calculation of the probability of completing the project within a given time period. A single critical path is thus calculated and relied upon, where in reality, there may be numerous possible critical paths that exist. For a large network plan the probability that any given path could be the critical path may be very small. PERT method yields results which are biased high. The construction project manager is thus grossly misled into thinking his chances are very good, when in reality they are very poor. If network has multiple parallel paths with relatively equal means, PERT calculations will be considerably biased [20]). As a result, the time to complete a project calculated by the traditional PERT method is almost always too short [21]).

Similar results have also been observed when using the technique of crashing PERT [22]). Completion times with the PERT method are much shorter than completion times calculated with the Monte Carlo method [15, 23, 24].

\section{Methods}

The problem of calculating a realistic duration for the completion of events is formulated as follows:

Activities $i-j$ and $i-k$ are performed in parallel (Fig. 1). Their late completion time defines the duration for the fulfilment of an event $m\left(t^{m}\right)$.

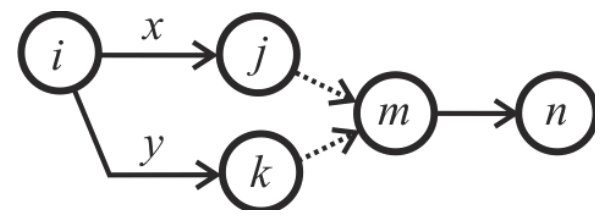

\section{Figure 1. The scheme for the calculation of $t^{m}$}

Durations of performance of works of $i-j$ and $i-k$ are independent random variables ( $X$ and $Y$ ). The durations are characterized by distributions $f_{1}(x)$ and $f_{2}(y)$.

The distribution of the function $Z$ in Equation 1 is established as:

$$
Z=\max (X, Y) \text {. }
$$

The problem is solved by using a universal DCl method (division, comparison, integration). The method is based on consecutive computing procedures listed as follows:

1. Parameters of the initial event of $i$ are established. For the first event, $\left(t_{i}=0\right)$.

2. The total area under a density curve is equal to 1 and divided by $p$, the number equal areas: $\left(S=\frac{1}{p}\right)$. The centre of gravity of each area is defined. These points will define $p$ as equiprobable values of duration $\left(x_{1}, x_{2} \ldots x_{p-1}, x_{p} ; y_{1}, y_{2} \ldots y_{p-1}, y_{p}\right)$ (Fig. 2).

Калугин Ю.Б. Универсальный метод вычисления достоверных сроков реализации проекта // Инженерно-строительный журнал. 2016. № 7(67). С. 70-80. 

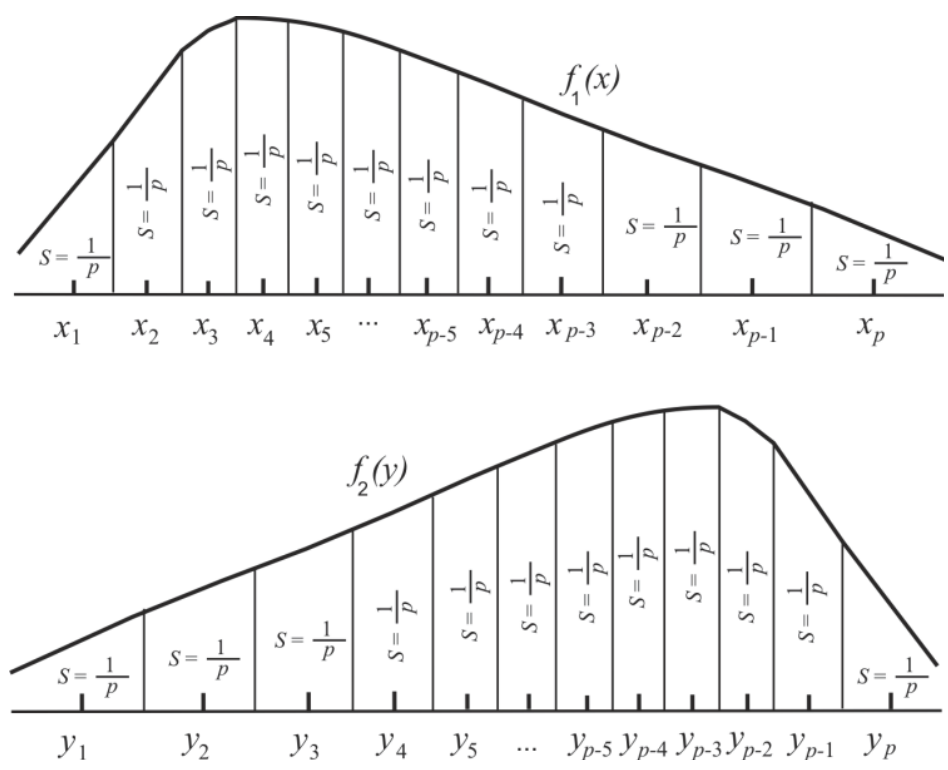

Figure 2. Distributions of activities (i-j, $i-k)$

3. Equiprobable durations for the fulfilment of events of $j$ are defined as:

$$
\begin{aligned}
& t_{1}^{j}=t^{i}+x_{1} ; \\
& t_{2}^{j}=t^{i}+x_{2} ; \\
& \cdots \\
& t_{p-1}^{j}=t^{i}+x_{p-1} ; \\
& t_{p}^{j}=t^{i}+x_{p} .
\end{aligned}
$$

4. Equiprobable durations for the fulfilment of events of $k$ are defined as:

$$
\begin{aligned}
& t_{1}^{k}=t^{i}+y_{1} ; \\
& t_{2}^{k}=t^{i}+y_{2} ; \\
& \cdots \\
& t_{p-1}^{k}=t^{i}+y_{p-1} ; \\
& t_{p}^{k}=t^{i}+y_{p} .
\end{aligned}
$$

5. Completion times for event $m$ are calculated. To this end, equiprobable durations for the fulfilment of an event $j$ are compared in pairs to the equiprobable durations for the fulfilment of an event $k$. In each pair, the maximum value is chosen.

$$
\begin{aligned}
& t_{1, r}^{m}=\max \left(t_{1}^{j} ; t_{r}^{k}\right), r=1 \ldots p ; \\
& t_{2, r}^{m}=\max \left(t_{2}^{j} ; t_{r}^{k}\right), r=1 \ldots p ; \\
& \ldots \\
& t_{p-1, r}^{m}=\max \left(t_{p-1}^{j} ; t_{r}^{k}\right), r=1 \ldots p ; \\
& t_{p, r}^{m}=\max \left(t_{p}^{j} ; t_{r}^{k}\right), r=1 \ldots p .
\end{aligned}
$$

The ascending numerical series is a result formed by $p^{2}$ of the equiprobable values of the durations for the fulfilment of an event $m\left(t_{1}^{m}, t_{2}^{m}, \ldots, t_{s}^{m}, \ldots, t_{p^{2}-1}^{m}, t_{p^{2}}^{m}\right), t_{s-1}^{m} \leq t_{s}^{m}, s=1 \ldots p^{2}$.

6. This series is consistently divided by $p$ segments for the purpose of further calculation reduction.

Kalugin Yu.B. Universal method for calculation of reliable completion times. Magazine of Civil Engineering. 2016. No. 7. Pp. 70-80. doi: 10.5862/MCE.67.7 
Each segment includes $p$ of values $\left(t_{1}^{m}, \ldots, t_{p}^{m} ; t_{p+1}^{m}, \ldots, t_{2 p}^{m} ; \ldots ; t_{(p-1) p}^{m}, \ldots, t_{p^{2}}^{m}\right)$. The integrated series $\left(t_{1}^{m}, t_{2}^{m}, \ldots, t_{p-1}^{m}, t_{p}^{m}\right)$ is formed by the mean values of each segment.

7. The equiprobable values of the duration of $m-n\left(t_{1}^{m n}, t_{2}^{m n}, \ldots, t_{p}^{m n}\right)$ are defined (similar to 2).

8. The equiprobable durations for the fulfilment of events $n$ are defined as,

$$
\begin{aligned}
& t_{11}^{n}=t_{1}^{m}+t_{1}^{m n} ; t_{12}^{n}=t_{1}^{m}+t_{2}^{m n} ; \ldots ; t_{1 p}^{n}=t_{1}^{m}+t_{p}^{m n} ; \\
& t_{21}^{n}=t_{2}^{m}+t_{1}^{m n} ; t_{22}^{n}=t_{2}^{m}+t_{2}^{m n} ; \ldots ; t_{2 p}^{n}=t_{2}^{m}+t_{p}^{m n} ; \\
& \ldots \\
& t_{(p-1) 1}^{n}=t_{p-1}^{m}+t_{1}^{m n} ; t_{(p-1) 2}^{n}=t_{p-1}^{m}+t_{2}^{m n} ; \ldots ; t_{(p-1) p}^{n}=t_{p-1}^{m}+t_{p}^{m n} ; \\
& t_{p 1}^{n}=t_{p}^{m}+t_{1}^{m n} ; t_{p 2}^{n}=t_{p}^{m}+t_{2}^{m n} ; \ldots ; t_{p p}^{n}+t_{p}^{m}+t_{p}^{m n} .
\end{aligned}
$$

The ascending numerical series is a result formed by $p^{2}$ of the equiprobable values of the durations for the fulfilment of an event $n\left(t_{1}^{n}, t_{2}^{n}, \ldots, t_{s}^{n}, \ldots, t_{p^{2}-1}^{n}, t_{p^{2}}^{n}\right), t_{s-1}^{n} \leq t_{s}^{n}, s=1 \ldots p^{2}$.

9. This series is consistently divided by $p$ segments for the purpose of further calculation reduction.

Each segment includes $p$ of values $\left(t_{1}^{n}, \ldots, t_{p}^{n} ; t_{p+1}^{n}, \ldots, t_{2 p}^{n} ; \ldots ; t_{(p-1) p}^{n}, \ldots, t_{p^{2}}^{n}\right)$. The integrated series $\left(t_{1}^{n}, t_{2}^{n}, \ldots, t_{p-1}^{n}, t_{p}^{n}\right)$ is formed by the mean values of each segment.

Calculations for two parallel works of equal duration and standard normal distribution [25] (for $p=2,4,8,10,12,14,16,32,64,128,256,512$ ) showed the following result:

\begin{tabular}{|c|c|c|c|c|c|c|c|c|c|c|c|c|c|}
\hline \multirow{2}{*}{\multicolumn{2}{|c|}{ Parameters }} & \multicolumn{12}{|c|}{$\boldsymbol{x}$} \\
\hline & & -1.7317 & -1.1503 & -0.8122 & -0.5485 & -0.3186 & -0.1046 & 0.1046 & 0.3186 & 0.5485 & 0.8122 & 1.1503 & 1.7317 \\
\hline & & \multicolumn{12}{|c|}{144 equiprobable values for the fulfilment of event $m$} \\
\hline \multirow{12}{*}{$\lambda$} & -1.7317 & -1.7317 & -1.1503 & -0.8122 & -0.5485 & -0.3186 & -0.1046 & 0.1046 & 0.3186 & 0.5485 & 0.8122 & 1.1503 & 1.7317 \\
\hline & -1.1503 & -1.1503 & -1.1503 & -0.8122 & -0.5485 & -0.3186 & -0.1046 & 0.1046 & 0.3186 & 0.5485 & 0.8122 & 1.1503 & 1.7317 \\
\hline & -0.8122 & -0.8122 & -0.8122 & -0.8122 & -0.5485 & -0.3186 & -0.1046 & 0.1046 & 0.3186 & 0.5485 & 0.8122 & 1.1503 & 1.7317 \\
\hline & -0.5485 & -0.5485 & -0.5485 & -0.5485 & -0.5485 & -0.3186 & -0.1046 & 0.1046 & 0.3186 & 0.5485 & 0.8122 & 1.1503 & 1.7317 \\
\hline & -0.3186 & -0.3186 & -0.3186 & -0.3186 & -0.3186 & -0.3186 & -0.1046 & 0.1046 & 0.3186 & 0.5485 & 0.8122 & 1.1503 & 1.7317 \\
\hline & -0.1046 & -0.1046 & -0.1046 & -0.1046 & -0.1046 & -0.1046 & -0.1046 & 0.1046 & 0.3186 & 0.5485 & 0.8122 & 1.1503 & 1.7317 \\
\hline & 0.1046 & 0.1046 & 0.1046 & 0.1046 & 0.1046 & 0.1046 & 0.1046 & 0.1046 & 0.3186 & 0.5485 & 0.8122 & 1.1503 & 1.7317 \\
\hline & 0.3186 & 0.3186 & 0.3186 & 0.3186 & 0.3186 & 0.3186 & 0.3186 & 0.3186 & 0.3186 & 0.5485 & 0.8122 & 1.1503 & 1.7317 \\
\hline & 0.5485 & 0.5485 & 0.5485 & 0.5485 & 0.5485 & 0.5485 & 0.5485 & 0.5485 & 0.5485 & 0.5485 & 0.8122 & 1.1503 & 1.7317 \\
\hline & 0.8122 & 0.8122 & 0.8122 & 0.8122 & 0.8122 & 0.8122 & 0.8122 & 0.8122 & 0.8122 & 0.8122 & 0.8122 & 1.1503 & 1.7317 \\
\hline & 1.1503 & 1.1503 & 1.1503 & 1.1503 & 1.1503 & 1.1503 & 1.1503 & 1.1503 & 1.1503 & 1.1503 & 1.1503 & 1.1503 & 1.7317 \\
\hline & 1.7317 & 1.7317 & 1.7317 & 1.7317 & 1.7317 & 1.7317 & 1.7317 & 1.7317 & 1.7317 & 1.7317 & 1.7317 & 1.7317 & 1.7317 \\
\hline \multicolumn{2}{|c|}{$\begin{array}{l}\text { Integrated series. } \\
\text { Mean }=0.54025\end{array}$} & -0.907 & -0.395 & -0.122 & 0.105 & 0.301 & 0.472 & 0.614 & 0.812 & 1.038 & 1.15 & 1.683 & 1.732 \\
\hline
\end{tabular}

The true mean value of the duration for the performance of an event $m$ did not exceed 0.56427 $\left(\mu^{*} \leq 0.56427\right)$. At $p=12, \mu^{12}=0.54025$. The deviation from the true value $\left(\mu^{*}\right)$ did not exceed $5 \%$. This indicates that this method is suitable for practical calculations of a network at $p=12$ (Table 1).

Table 1. Calculated equiprobable values for the fulfilment of event $\boldsymbol{m}$

Калугин Ю.Б. Универсальный метод вычисления достоверных сроков реализации проекта // Инженерно-строительный журнал. 2016. № 7(67). С. 70-80. 
The stated method used Microsoft Excel.

The distribution of parameters $\mathrm{X}(\mathrm{Y}), \mathrm{Z}$ (for events $j(k), m)$ is presented in Figure 3. intervals).

Density was defined as the difference of adjacent equiprobable values (at half values for extreme

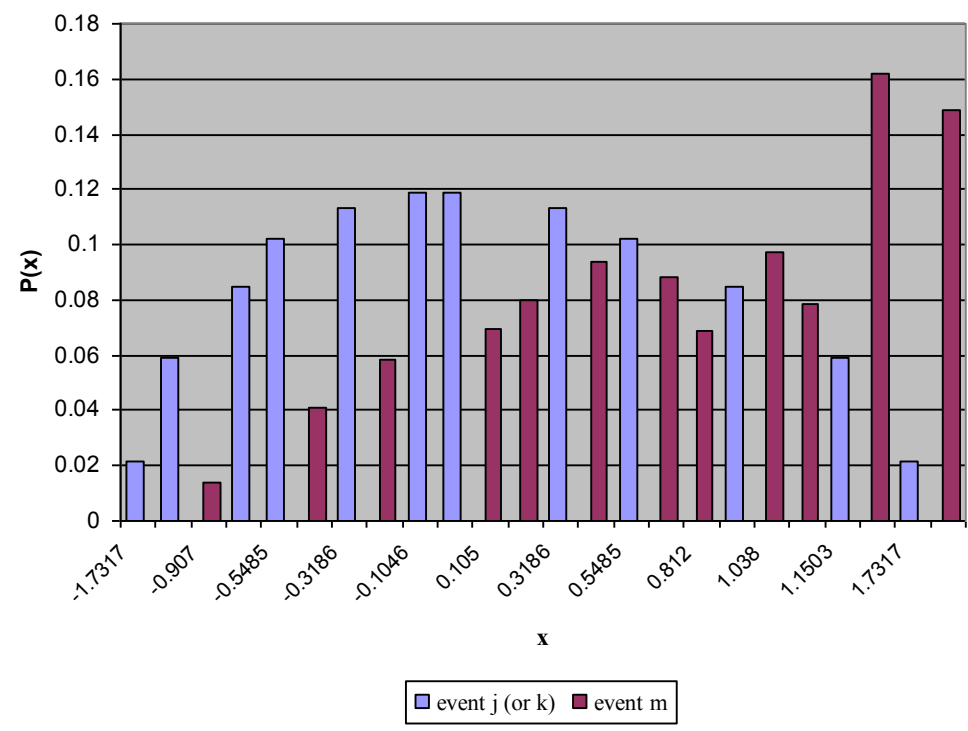

Figure 3. Distribution of parameters $X(Y), Z$ (for events $j(k), m)$

Calculations show a shift of the maximum density to the maximum values $Z$.

\section{Results and Discussion}

The universal method presented in this paper was used to calculate a more realistic duration for the construction of a banquette of the road.

The technology and the organization of works are defined in the flow sheet [26]. According to the flow sheet the work process is performed in five divisions during one shift (Fig. 4).

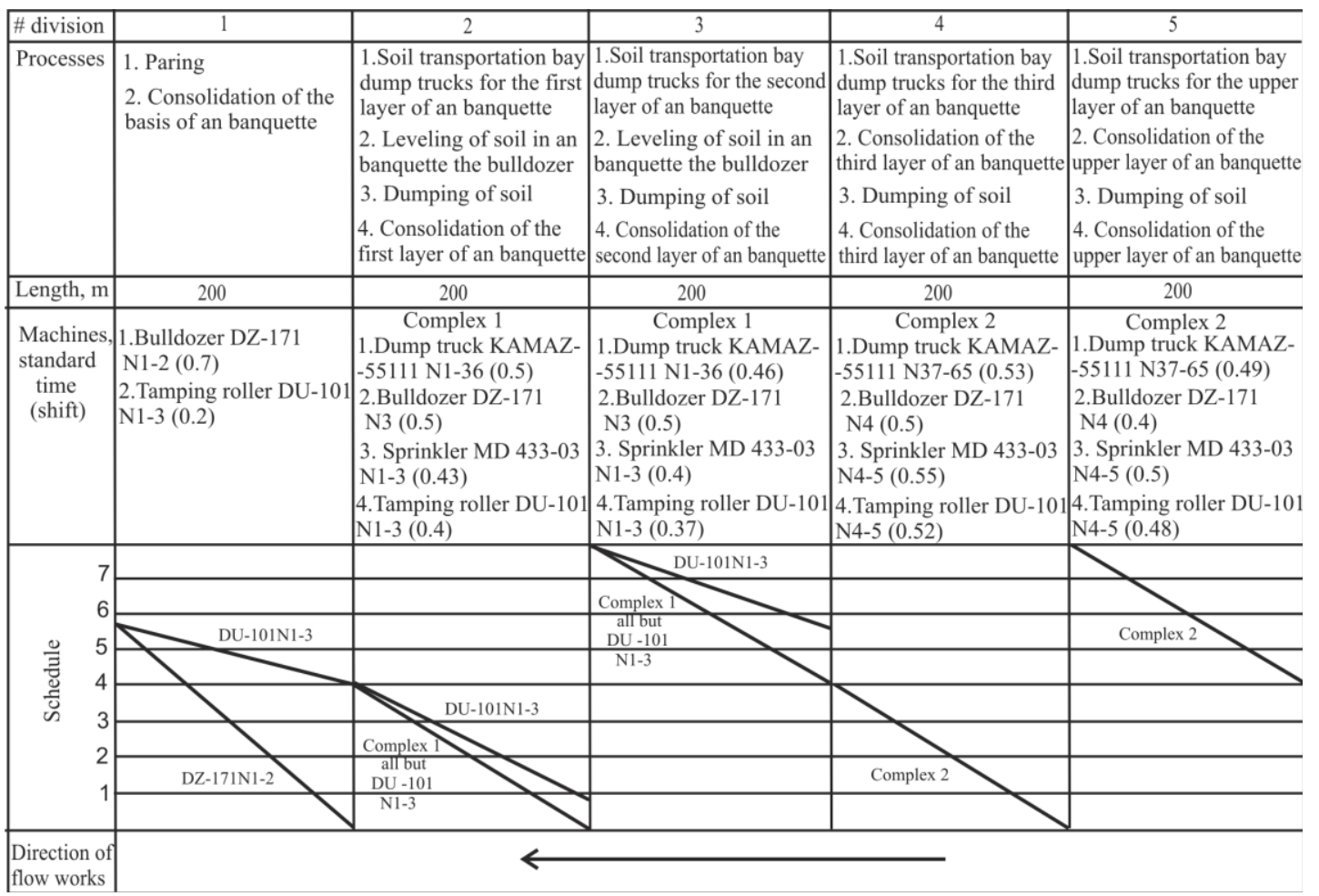

Figure 4. Fragment of flow sheet for the construction of a banquette

Kalugin Yu.B. Universal method for calculation of reliable completion times. Magazine of Civil Engineering. 2016. No. 7. Pp. 70-80. doi: 10.5862/MCE.67.7 
The network schedule was composed on the basis of the flow sheet (Fig. 5).

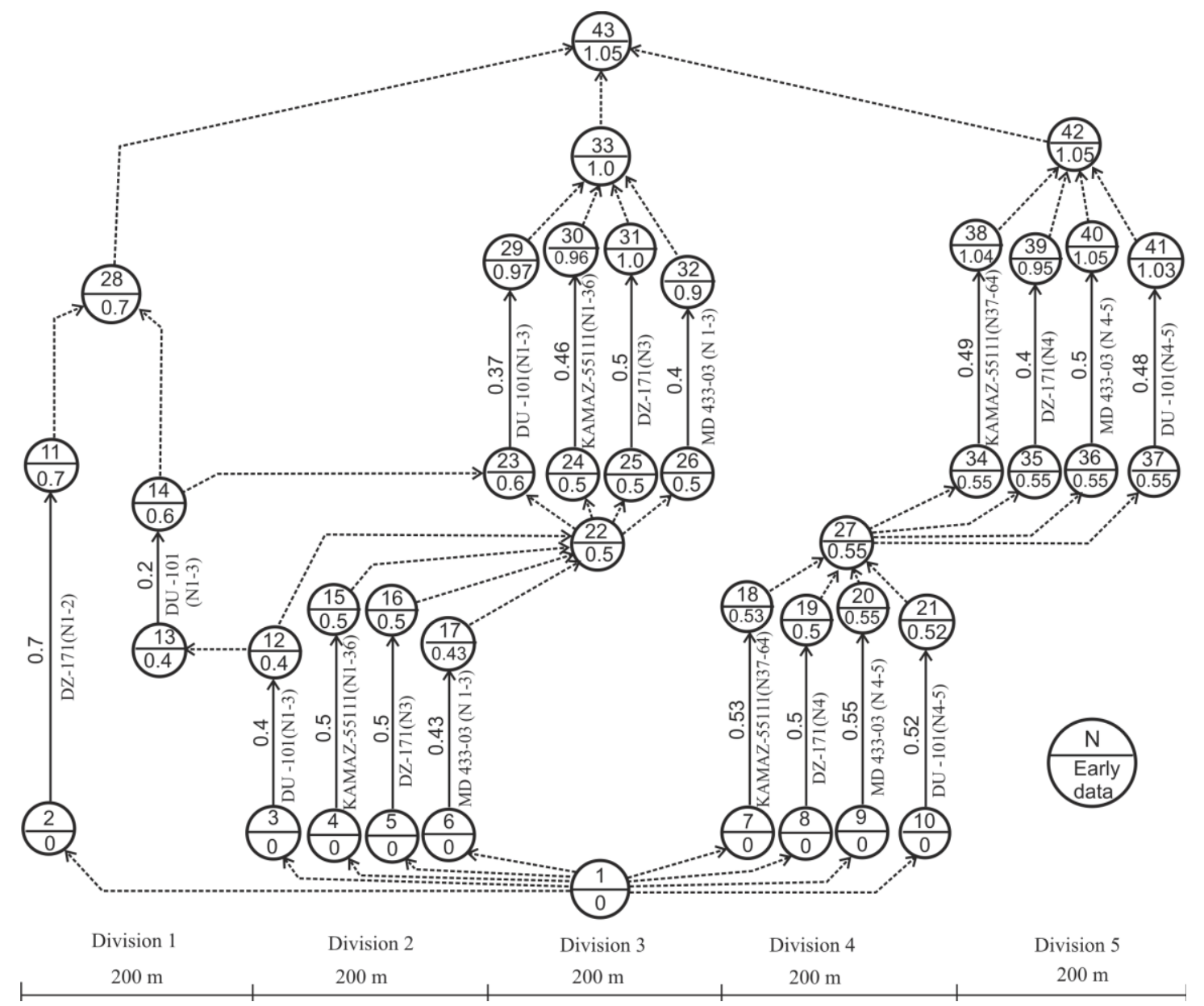

Figure 5. Network schedule

The critical path (activities 1-9-20-27-36-40-42-43) is equal to 1.05 shifts.

The stochastic parameters of the equipment [10] are presented in Table 2.

Table 2. Stochastic parameters of equipment

\begin{tabular}{|c|c|c|c|c|c|c|c|c|c|c|c|c|c|}
\hline Machines & \multicolumn{9}{|c|}{12 equiprobable values standard time, shift } & $\bar{t}$ \\
\hline Bulldozers & 0.644 & 0.719 & 0.772 & 0.817 & 0.862 & 0.909 & 0.960 & 1.018 & 1.089 & 1.184 & 1.332 & 1.698 & 1.00 \\
\hline $\begin{array}{c}\text { Tamping } \\
\text { rollers }\end{array}$ & 0.655 & 0.729 & 0.780 & 0.826 & 0.870 & 0.915 & 0.964 & 1.020 & 1.088 & 1.178 & 1.318 & 1.656 & 1.00 \\
\hline $\begin{array}{c}\text { Dump } \\
\text { rucks }\end{array}$ & 0.691 & 0.761 & 0.809 & 0.851 & 0.891 & 0.932 & 0.976 & 1.026 & 1.085 & 1.161 & 1.277 & 1.541 & 1.00 \\
\hline \begin{tabular}{c} 
Sprinklers \\
\hline
\end{tabular} & 0.843 & 0.887 & 0.915 & 0.939 & 0.959 & 0.979 & 1.001 & 1.023 & 1.048 & 1.078 & 1.120 & 1.200 & 1.00 \\
\hline
\end{tabular}

The stochastic parameters of the activities network schedule are presented in Table 3.

Калугин Ю.Б. Универсальный метод вычисления достоверных сроков реализации проекта // Инженерно-строительный журнал. 2016. № 7(67). С. 70-80. 
Table 3. Stochastic parameters of processes of construction of banquette

\begin{tabular}{|c|c|c|c|c|c|c|c|c|c|c|c|c|c|c|}
\hline \multirow{3}{*}{\begin{tabular}{|c|}
$\begin{array}{c}\# \\
\text { activity }\end{array}$ \\
$2-11$ \\
\end{tabular}} & Processes & & \multicolumn{11}{|c|}{12 equiprobable values time, shift } & $\bar{t}$ \\
\hline & \multicolumn{14}{|c|}{ Division 1} \\
\hline & 1.ParingDZ-171 N1-2 & 0.451 & 0.503 & 0.540 & 0.572 & 0.603 & 0.636 & 0.672 & 0.712 & 0.762 & 0.828 & 0.932 & 1.188 & 0.700 \\
\hline $13-14$ & $\begin{array}{l}\text { 2. Consolidation of the } \\
\text { basis DU-101 N1-3 }\end{array}$ & 0.131 & 0.146 & 0.156 & 0.165 & 0.174 & 0.183 & 0.193 & 0.204 & 0.218 & 0.236 & 0.264 & 0.331 & 0.200 \\
\hline \multicolumn{15}{|c|}{ Division 2} \\
\hline $4-15$ & $\begin{array}{l}\text { 1. Soil transportation } \\
\text { KAMAZ-55111 } \\
\text { N1-36 }\end{array}$ & 0.346 & 0.381 & 0.405 & 0.426 & 0.446 & 0.466 & 0.488 & 0.513 & 0.543 & 0.581 & 0.639 & 0.771 & 0.500 \\
\hline $5-16$ & $\begin{array}{l}\text { 2.Leveling of soil DZ-171 } \\
\text { N3 }\end{array}$ & 0.322 & 0.359 & 0.386 & 0.408 & 0.431 & 0.454 & 0.480 & 0.509 & 0.544 & 0.592 & 0.666 & 0.849 & 0.500 \\
\hline $6-17$ & $\begin{array}{l}\text { 3. Dumping of soil MD } \\
433-03 \mathrm{~N} 1-3\end{array}$ & 0.362 & 0.381 & 0.393 & 0.404 & 0.413 & 0.421 & 0.430 & 0.440 & 0.450 & 0.464 & 0.482 & 0.516 & 0.430 \\
\hline $3-12$ & $\begin{array}{l}\text { 4. Consolidation of the } \\
\text { first layer DU-101 N1-3 }\end{array}$ & 0.262 & 0.292 & 0.312 & 0.330 & 0.348 & 0.366 & 0.386 & 0.408 & 0.435 & 0.471 & 0.527 & 0.662 & 0.400 \\
\hline \multicolumn{15}{|c|}{ Division 3} \\
\hline $24-30$ & $\begin{array}{l}\text { 1. Soil transportation } \\
\text { KAMAZ-55111 N1-36 }\end{array}$ & 0.318 & 0.350 & 0.372 & 0.391 & 0.410 & 0.429 & 0.449 & 0.472 & 0.499 & 0.534 & 0.587 & 0.709 & 0.460 \\
\hline $25-31$ & $\begin{array}{l}\text { 2.Leveling of soil } \\
\text { DZ-171 N3 }\end{array}$ & 0.322 & 0.359 & 0.386 & 0.408 & 0.431 & 0.454 & 0.480 & 0.509 & 0.544 & 0.592 & 0.666 & 0.849 & 0.500 \\
\hline $26-32$ & $\begin{array}{l}\text { 3. Dumping of soil MD } \\
433-03 \text { N1-3 }\end{array}$ & 0.337 & 0.355 & 0.366 & 0.375 & 0.384 & 0.392 & 0.400 & 0.409 & 0.419 & 0.431 & 0.448 & 0.480 & 0.400 \\
\hline $23-29$ & $\begin{array}{l}\text { 4. Consolidation of the } \\
\text { second layer } \\
\text { DU-101 N1-3 }\end{array}$ & 0.242 & 0.270 & 0.289 & 0.306 & 0.322 & 0.339 & 0.357 & 0.377 & 0.403 & 0.436 & 0.488 & 0.613 & 0.370 \\
\hline \multicolumn{15}{|c|}{ Division 4} \\
\hline $7-18$ & $\begin{array}{l}\text { 1. Soil transportation } \\
\text { KAMAZ-55111 } \\
\text { N37-64 }\end{array}$ & 0.366 & 0.403 & 0.429 & 0.451 & 0.472 & 0.494 & 0.517 & 0.544 & 0.575 & 0.615 & 0.677 & 0.817 & 0.530 \\
\hline 8-19 & $\begin{array}{l}\text { 2.Leveling of soil } \\
\text { DZ-171 N4 }\end{array}$ & 0.322 & 0.359 & 0.386 & 0.408 & 0.431 & 0.454 & 0.480 & 0.509 & 0.544 & 0.592 & 0.666 & 0.849 & 0.500 \\
\hline $9-20$ & $\begin{array}{l}\text { 3. Dumping of soil MD } \\
433-03 \text { N4-5 }\end{array}$ & 0.464 & 0.488 & 0.503 & 0.516 & 0.528 & 0.539 & 0.551 & 0.563 & 0.576 & 0.593 & 0.616 & 0.660 & 0.550 \\
\hline $10-21$ & $\begin{array}{l}\text { 4. Consolidation of the } \\
\text { third layer DU-101 N4-5 }\end{array}$ & 0.341 & 0.379 & 0.406 & 0.430 & 0.452 & 0.476 & 0.501 & 0.530 & 0.566 & 0.613 & 0.685 & 0.861 & 0.520 \\
\hline \multicolumn{15}{|c|}{ Division 5} \\
\hline $34-38$ & $\begin{array}{l}\text { 1. Soil transportation } \\
\text { KAMAZ-55111 } \\
\text { N37-64 }\end{array}$ & 0.339 & 0.373 & 0.396 & 0.417 & 0.437 & 0.457 & 0.478 & 0.503 & 0.532 & 0.569 & 0.626 & 0.755 & 0.490 \\
\hline 35-39 & $\begin{array}{l}\text { 2.Leveling of soil DZ-171 } \\
\text { N4 }\end{array}$ & 0.258 & 0.288 & 0.309 & 0.327 & 0.345 & 0.364 & 0.384 & 0.407 & 0.435 & 0.474 & 0.533 & 0.679 & 0.400 \\
\hline $36-40$ & $\begin{array}{l}\text { 3. Dumping of soil MD } \\
433-03 \text { N4-5 }\end{array}$ & 0.422 & 0.444 & 0.457 & 0.469 & 0.480 & 0.490 & 0.500 & 0.511 & 0.524 & 0.539 & 0.560 & 0.600 & 0.500 \\
\hline $37-41$ & $\begin{array}{l}\text { 4. Consolidation of the } \\
\text { upper layer DU-101 N4-5 }\end{array}$ & 0.314 & 0.350 & 0.374 & 0.396 & 0.418 & 0.439 & 0.463 & 0.490 & 0.522 & 0.565 & 0.633 & 0.795 & 0.480 \\
\hline
\end{tabular}

The stochastic parameters of the events network schedule were calculated by the universal method (Table 4).

Kalugin Yu.B. Universal method for calculation of reliable completion times. Magazine of Civil Engineering. 2016. No. 7. Pp. 70-80. doi: 10.5862/MCE.67.7 
Table 4. Stochastic parameters of events

\begin{tabular}{|c|c|c|c|c|c|c|c|c|c|c|c|c|c|c|c|}
\hline \multirow{2}{*}{$\begin{array}{c}\begin{array}{c}\# \\
\text { an of } \\
\text { event }\end{array} \\
1 \\
\end{array}$} & \multicolumn{12}{|c|}{12 equiprobable values time, shift } & \multirow{2}{*}{\begin{tabular}{|l|}
$\mathbf{P}(\mathbf{t})=$ \\
$\mathbf{0 . 2 5}$ \\
0.000 \\
\end{tabular}} & \multirow{2}{*}{$\begin{array}{c}P(t)= \\
0.5 \\
0.000 \\
\end{array}$} & \multirow{2}{*}{\begin{tabular}{|c|}
$P(t)=$ \\
0.75 \\
0.000 \\
\end{tabular}} \\
\hline & 0.000 & 0.000 & 0.000 & 0.000 & 0.000 & 0.000 & 0.000 & 0.000 & 0.000 & 0.000 & 0.000 & 0.000 & & & \\
\hline 2 & 0.000 & 0.000 & 0.000 & 0.000 & 0.000 & 0.000 & 0.000 & 0.000 & 0.000 & 0.000 & 0.000 & 0.000 & 0.000 & 0.000 & 0.000 \\
\hline 3 & 0.000 & 0.000 & 0.000 & 0.000 & 0.000 & 0.000 & 0.000 & 0.000 & 0.000 & 0.000 & 0.000 & 0.000 & 0.000 & 0.000 & 0.000 \\
\hline 4 & 000 & 0.000 & 0.000 & 000 & 0.000 & 0.000 & 0.000 & 0.000 & 0.000 & 0.000 & 0.000 & 0.000 & 0.000 & 0.000 & 000 \\
\hline 5 & 00 & 000 & 000 & 000 & 0.000 & 0.000 & 0.000 & 0.000 & 0.000 & 0.000 & 0.000 & 0.000 & 0.000 & 0.000 & 0.000 \\
\hline 6 & 00 & 0.000 & 0.000 & 0.000 & 0.000 & 0.000 & 0.000 & 0.000 & 0.000 & 0.000 & 0.000 & 0.000 & 0.000 & 0.000 & 000 \\
\hline 7 & 0 & 000 & 0.000 & 0 & 0.000 & 0.000 & 0.000 & 0.000 & 0.000 & 0.000 & 0.000 & 0.000 & 0.000 & 0.000 & 0.000 \\
\hline 8 & 0.000 & 0.000 & 0.000 & 0.000 & 0.000 & 0.000 & 0.000 & 0.000 & 0.000 & 0.000 & 0.000 & 0.000 & 0.000 & 0.000 & 000 \\
\hline 9 & 00 & 000 & 000 & 00 & 0.000 & 0.000 & 0.000 & 0.000 & 0.000 & 0.000 & 0.000 & 0.000 & 0.000 & 0.000 & 000 \\
\hline 10 & 0.000 & 0.000 & 0.000 & 0.000 & 0.000 & 0.000 & 0.000 & 0.000 & 0.000 & 0.000 & 0.000 & 0.000 & 0.000 & 0.000 & .000 \\
\hline 11 & & 3 & 0 & & & & & & & 0 & 41 & 80 & 0.556 & 0.667 & 795 \\
\hline 12 & 0.262 & 0.292 & 0.312 & 0.330 & 0.348 & 0.366 & 0.386 & 0.408 & 0.435 & 0.471 & 0.479 & 0.501 & 0.321 & 0.383 & 0.453 \\
\hline 13 & 62 & 0 & 0 & & & & & & & & & & 321 & 0.383 & 453 \\
\hline 14 & 0.433 & 0.473 & 0.500 & 0.523 & 0.544 & 0.567 & 0.591 & 0.617 & 0.648 & 0.687 & 0.702 & 0.728 & 0.511 & 0.584 & 0.667 \\
\hline 15 & 6 & 0.381 & 0.405 & 6 & 0.446 & 0.466 & 0.488 & 0.513 & 0.543 & 0.581 & 0.593 & 0.617 & 0.416 & 0.484 & 562 \\
\hline 16 & 22 & 359 & 386 & 08 & 0.431 & 0.454 & 0.480 & 0.509 & 0.544 & 0.592 & 0.601 & 0.629 & 0.397 & 0.476 & .568 \\
\hline 17 & 62 & 381 & 393 & 4 & 0 & 0.421 & 0.430 & 0.440 & 0.450 & 0.464 & 0.473 & 0.484 & 0.399 & 0.426 & 0.457 \\
\hline 18 & 0.366 & 403 & 0.429 & 0.451 & 0.472 & 0.494 & 0.517 & 0.544 & 0.575 & 0.615 & 0.628 & 0.654 & 0.440 & 0.512 & 595 \\
\hline 19 & 0.322 & 0.359 & 0.386 & 0.408 & 0.431 & 0.454 & 0.480 & 0.509 & 0.544 & 0.592 & 0.601 & 0.629 & 0.397 & 0.476 & 0.568 \\
\hline 20 & 0.464 & 488 & 0.503 & 0.516 & 0.528 & 0.539 & 0.551 & 0.563 & 0.576 & 0.593 & 0.605 & 0.618 & 0.510 & 0.545 & 585 \\
\hline 21 & 0.341 & 379 & 0.406 & 0.430 & 0.452 & 0.476 & 0.501 & 0.530 & 0.566 & 0.613 & 0.623 & 0.651 & 0.418 & 0.497 & 590 \\
\hline 22 & 0. & 60 & 83 & 6 & 24 & 0.542 & 0.577 & 0.611 & 0.650 & 0.665 & 0.689 & 0.716 & 0.495 & 0.571 & 0.657 \\
\hline 23 & 0.485 & 0.530 & 6 & & 1 & & & 0 & 30 & 0 & 0. & 0 & 570 & 0.651 & 45 \\
\hline 24 & 0. & 160 & 483 & 0 & 0.524 & 0.542 & 0.577 & 0.611 & 0.650 & 0.665 & 0.689 & 0.716 & 0.495 & 0.571 & 0.657 \\
\hline 25 & 6 & 0.460 & 3 & 0 & 0. & 0 & 0. & 0 & 0 & 0. & 0 & 0 & 95 & 0.571 & 657 \\
\hline 26 & 0.426 & 0.460 & 0.483 & 0.506 & 0.524 & 0.542 & 0.577 & 0.611 & 0.650 & 0.665 & 0.689 & 0.716 & 0.495 & 0.571 & 0.657 \\
\hline 27 & 0.505 & 536 & 560 & 581 & 0 & 0.616 & 0.644 & 0.666 & 0.682 & 0.785 & 0.760 & 0.786 & 0.571 & 0.643 & 0.734 \\
\hline 28 & 0.512 & 564 & 0.600 & 0.630 & 0.660 & 0.687 & 0.725 & 0.758 & 0.812 & 0.861 & 0.880 & 0.916 & 0.615 & 0.717 & 0.836 \\
\hline 29 & 0.793 & 0.859 & 0.899 & 0.932 & 0.965 & 0.998 & 1.032 & 1.072 & 1.115 & 1.159 & 1.192 & 1.230 & 0.915 & 1.020 & 1.137 \\
\hline 30 & 0.808 & 0.869 & 0.908 & 0.943 & 0.975 & 1.009 & 1.043 & 1.083 & 1.129 & 1.184 & 1.209 & 1.248 & 0.926 & 1.034 & 1.157 \\
\hline 31 & 0.817 & 0.884 & 0.927 & 0.967 & 1.003 & 1.040 & 1.080 & 1.123 & 1.175 & 1.239 & 1.265 & 1.308 & 0.947 & 1.069 & 1.207 \\
\hline 32 & 0.807 & 350 & 78 & 2 & 0.925 & 0.951 & 0.980 & 1.011 & 1.043 & 1.087 & 1.103 & 1.132 & 0.890 & 0.973 & 065 \\
\hline 33 & 0.990 & 1.056 & 1.098 & 1.137 & 1.164 & 1.194 & 1.230 & 1.249 & 1.295 & 1.325 & 1.365 & 1.400 & 1.118 & 1.209 & 1.310 \\
\hline 34 & 5 & 6 & 0 & & 9 & 6 & 4 & 6 & 32 & 0. & 0. & 0.786 & 0.571 & 0.643 & 734 \\
\hline 35 & 0.505 & 0.536 & 0.560 & 0.581 & 0.599 & 0.616 & 0.644 & 0.666 & 0.682 & 0.785 & 0.760 & 0.786 & 0.571 & 0.643 & 0.734 \\
\hline 36 & 0.505 & 36 & 0 & 81 & 9 & 6 & 4 & 66 & 0.682 & 0.785 & 0.760 & 0.786 & 0.571 & 0.643 & 734 \\
\hline 37 & 0.505 & 0.536 & 0.560 & 0.581 & 0.599 & 0.616 & 0.644 & 0.666 & 0.682 & 0.785 & 0.760 & 0.786 & 0.571 & 0.643 & 0.734 \\
\hline 38 & 0.908 & 0.970 & 1.008 & 1. & 1.072 & 1.108 & 1.144 & 1.187 & 1.233 & 1.283 & 1.311 & 1.350 & 1.024 & 1.134 & 1.258 \\
\hline 39 & 0.823 & 0.882 & 0.918 & 0.950 & 0.980 & 1.014 & 1.051 & 1.095 & 1.142 & 1.193 & 1.217 & 1.256 & 0.934 & 1.043 & 1.167 \\
\hline 40 & 0.976 & 1.022 & 1.051 & 1.076 & 1.098 & 1.121 & 1.145 & 1.173 & 1.216 & 1.276 & 1.279 & 1.308 & 1.063 & 1.145 & 1.246 \\
\hline 41 & 0.885 & 0.948 & 0.988 & 1.022 & 1.056 & 1.092 & 1.133 & 1.175 & 1.223 & 1.278 & 1.306 & 1.347 & 1.005 & 1.121 & 1.250 \\
\hline 42 & 1.082 & 1.151 & 1.187 & 1.222 & 1.256 & 1.282 & 1.312 & 1.341 & 1.366 & 1.391 & 1.437 & 1.470 & 1.204 & 1.291 & 1.378 \\
\hline 43 & 1.145 & 1.206 & 1.245 & 1.273 & 1.303 & 1.324 & 1.349 & 1.369 & 1.386 & 1.450 & 1.468 & 1.497 & 1.259 & 1.335 & 1.418 \\
\hline
\end{tabular}

Калугин Ю.Б. Универсальный метод вычисления достоверных сроков реализации проекта // Инженерно-строительный журнал. 2016. № 7(67). С. 70-80. 
The distribution of completion times for the 43rd event is presented in Figure 6.

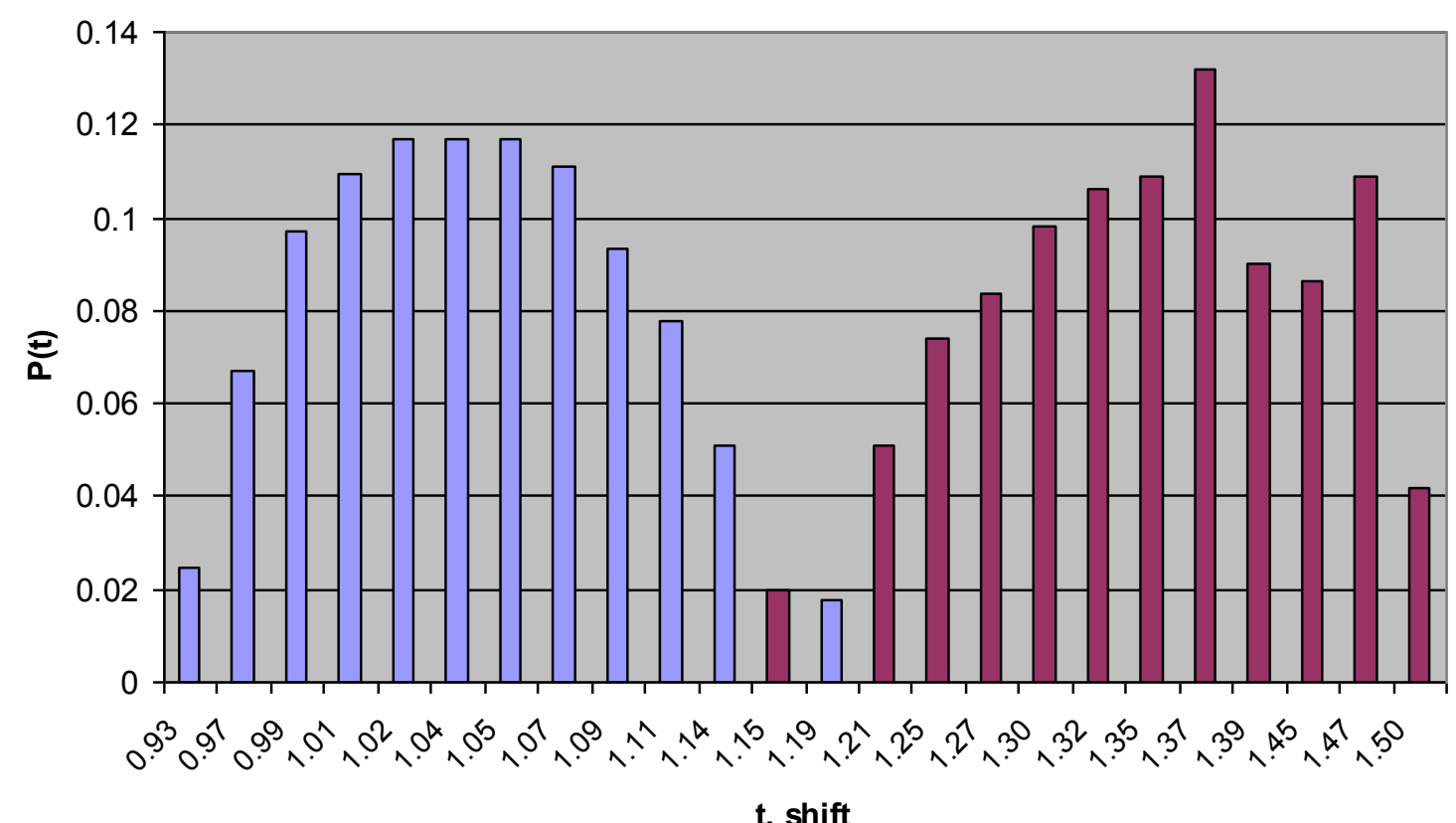

$\square$ method PERT $\square$ Universal method

Figure 6. Distribution of completion times (for the 43rd event)

The duration of the calculations lasted 3-4 s.

Using a known critical path method, the mean duration was equal to 1.05 shifts, while the mean duration was equal to 1.335 shifts with the universal method. With a probability of 0.75 , construction works was scheduled to end after 1.418 shifts

\section{Conclusions}

1. The actual duration of various construction projects significantly exceeds the scheduled durations. The reason for the significant difference between planned and actual durations is, primarily, the impact of the numerous stochastic factors on the works. Additionally, the traditional PERT method uses a single critical path. As a result, the PERT method of calculating the time to complete a project is almost always too short.

The construction project manager is thus grossly misled into thinking his chances are very good.

2. The universal method to calculate an accurate duration for the fulfilment of events is presented. The basics of this universal $\mathrm{DCl}$ method (division, comparison, integration) include:

- The total area under a density curve (for parallel activities with a joint event) divides into $p$ equal areas. The centres of gravity of each area will define $p$ as equiprobable values of duration;

- Equiprobable completion times of one activity are compared in pairs to completion times of another activity. In each pair the maximum value is chosen. The ascending numerical series as a result is formed by the $p^{2}$ of the equiprobable values of the duration for the fulfilment of an event;

- This series is consistently divided by $p$ segments for the purpose of further calculation reduction. The integrated series is formed by the mean value of each segment.

Calculations for two parallel works of equal duration and standard normal distribution showed that this method is suitable for practical calculations of a network at $p=12$

The method presented in this study used Microsoft Excel.

3. The universal $\mathrm{DCl}$ method was used to calculate a realistic duration for the construction of a banquette of the road. The technology and the organization of works are defined in the flow sheet. According to the flow sheet, the works will be performed in five divisions during 1.05 shifts. With a traditional PERT method, the mean duration is also equal to 1.05 shifts. Using the universal DCI method, Kalugin Yu.B. Universal method for calculation of reliable completion times. Magazine of Civil Engineering. 2016. No. 7. Pp. 70-80. doi: 10.5862/MCE.67.7 
the mean duration is equal to 1.34 shifts; however, with a probability of 0.75 included, works will end after 1.42 shifts. The calculations lasted some seconds.

4. These results show the efficacy of the universal $\mathrm{DCl}$ method to calculate more realistic completion times. This method considers an increase in the actual completion times in comparison with the planned completion times (using the PERT method), especially since in reality, numerous possible critical paths may exist.

The suggested $\mathrm{DCl}$ method can be recommended for use by construction project managers in order to prevent a potential failure of project completion deadlines.

\section{References}

1. Afanasyev V., Afanasyev A. Potochnaya organizatsiya rabot $v$ stroitelstve [Flowing organization works in construction]. Saint-Petersburg: SPbGASU. 2000. 169 p. (rus)

2. Bolotin S.; Birjukov A. Time management in drafting probability schedules for construction work. World Applied Sciences Journal. 2013. Vol. 23. No. 13. Pp. 1-4.

3. Bovteev S.V., Kanyukova S.V. Development of methodology for time management of construction projects. Magazine of Civil Engineering. 2016. No. 2(62). Pp. 102-112.

4. Sadi A., Sadiq A.-H. Causes of delay in large construction projects. Journal Project Management. 2006. No. 4(24). Pp. 349-357.

5. Simankina T., Sergeenkova O. Resource optimization of construction on a basis of the frontal and rhythmical stream. Applied Mechanics and Materials. 2015. Vols. 725-726. Pp. 984-989.

6. Trauner T., Manginelli W., Lowe J., Nagata M., Furniss B. Construction Delays: Understanding Them Clearly and Delay Analysis in Construction Analyzing Them Correctly. London, UK: Elsevier Inc. 2009. 288 p.

7. Kalugin Yu.B. Modelirovanie vozdeistviya destabiliziruyushchikh faktorov na tekhnologicheskii kompleks [Modeling of destabilizing factors of the technological complex]. News of Higher Educational Institutions. Construction. 2013. No. 7. Pp. 93-102. (rus)

8. Golenko-Ginzburg D.I. Stokhasticheskie setevye modeli planirovaniya $i$ upravleniya razrabotkami [Stochastic models of network planning and management development]. Voronezh: Science Book. 2010. 284 p. (rus)

9. Golenko-Ginzburg D. On the distribution of activity time in PERT. Journal of the Operational Research Society. 1988. Vol. 39. No. 8. Pp. 767-771.

10. Maltsev Yu.A. Ekonomiko-matematicheskie metody proektirovaniya transportnykh sooruzhenii [Economicmathematical methods of design of transport objects]. Moscow: Academia. 2010. 320 p. (rus)

11. Miller R. Schedule, Cost and Profit Control with PERT. McGraw-Hill. 1963. 58 p.

12. Rukovodstvo po razrabotke i primeneniyu veroyatnostnykh setevykh modelei $v$ stroitel'stve [Guidelines for the development and application of probabilistic network models in construction]. Moscow: TsNIPIASS. 1979. 56 p. (rus)

13. A Guide to the Project Management Body of Knowledge. Project Management Institute. 2008. 506 p.

14. Birrell G. Construction planning beyond the critical path. Journal of Construction Division ASCE. 1980. No. 3(106). Pp. 389-407.

15. Golenko D.I. Statisticheskie metody setevogo planirovaniya $i$ upravleniya [Statistical methods of network planning and management]. Moscow: Nauka. 1968. 400 p. (rus)

16. Mubarak S. Construction Project Scheduling and Control.

\section{Литература}

1. Афранасьев В.А., Афанасьев А.В. Поточная организация работ в строительстве. СПб: СПбГАСУ. 169 c.

2. Bolotin S.; Birjukov A. Time management in drafting probability schedules for construction work // World Applied Sciences Journal. 2013. Vol. 23. № 13. Pp. 1-4.

3. Бовтеев С.В., Канюкова С.В. Развитие методики контроля сроков инвестиционно-строительного проекта // Инженерно-строительный журнал. 2016. № 2(62). C. 102-112.

4. Sadi A.A. Sadiq A.-H. Causes of delay in large construction projects // Journal Project Management. 2006. № 4(24). Pp. 349-357.

5. Simankina T., Sergeenkova O. Resource optimization of construction on a basis of the frontal and rhythmical stream // Applied Mechanics and Materials. 2015. Vols. 725-726. Pp. 984-989.

6. Trauner T., Manginelli W., Lowe J., Nagata M., Furniss B. Construction Delays: Understanding Them Clearly and Delay Analysis in Construction Analyzing Them Correctly. London, UK: Elsevier Inc. 2009. 288 p.

7. Калугин Ю.Б. Моделирование воздействия дестабилизирующих факторов на технологический комплекс // Известия вузов. Строительство. 2013. № 7. С. 93-102.

8. Голенко-Гинзбург Д.И. Стохастические сетевые модели планирования и управления разработками. Воронеж: Научная книга. 2010. 284 с.

9. Golenko-Ginzburg D. On the distribution of activity time in PERT // Journal of the Operational Research Society. 1988. Vol. 39. № 8. Pp. 767-771.

10. Мальцев Ю.А. Экономико-математические методы проектирования транспортных сооружений. М.: Академия. 2010. 320 с.

11. Miller R. Schedule, Cost and Profit Control with PERT. McGraw-Hill. 1963. 58 p.

12. Руководство по разработке и применению вероятностных сетевых моделей в строительстве. М.: ЦНИПИАСС. 1979. 56 с.

13. A Guide to the Project Management Body of Knowledge. Project Management Institute. 2008. 506 p.

14. Birrell G. Construction planning beyond the critical path // Journal of Construction Division ASCE. 1980. № 3(106). Рp. 389-407.

15. Голенко Д.И. Статистические методы сетевого планирования и управления. М.: Наука. 1968. 400 с.

16. Mubarak S. Construction Project Scheduling and Control. Second Edition. New Jersey. Hoboken: John Wiley \& Sons, Inc. 2010. 479 p.

17. Олейникова C.A. Критический анализ метода PERT решения задачи управления проектами со случайной длительностью выполнения работ // Системы управления и информационные технологии. 2013. Т. 51. №1. C. 20-24.

18. Herroelen W., Leus R. Project scheduling under Калугин Ю.Б. Универсальный метод вычисления достоверных сроков реализации проекта // Инженерно-строительный журнал. 2016. № 7(67). С. 70-80. 
Second Edition. New Jersey. Hoboken: John Wiley \& Sons, Inc. 2010. 479 p.

17. Oleinikova S.A. Kriticheskii analiz metoda PERT resheniya zadachi upravleniya proektami so sluchainoi dlitel'nost'yu vypolneniya rabot [Critical analysis of the PERT method for solving the problem of project management with random duration of the work]. Control Systems and Information Technology. 2013. Vol. 51. No. 1. Pp. 20-24. (rus)

18. Herroelen W., Leus R. Project scheduling under uncertainty: Survey and research potentials. European Journal of Operational Research. 2005. No. 165. Pp. 289306.

19. Liberatore M. Critical path analysis with fuzzy activity times. Journal of Transactions on Engineering Management. 2008. Vol. 55. No. 2. Pp. 329-337.

20. Klingel A. Bias in PERT completion times calculations for a real network. Management Science. 1966. Vol. 13. No. 4. Pp. 476-489.

21. Schonberger R. Why projects are always late: a rationale based on manual simulation of a PERT/CPM network. Interfaces. 1981. Vol. 11. No. 5. Pp. 66-70.

22. Haga W., O'Keefe T. Crashing PERT networks: A simulation approach. Proc. of the 4th International conference of the Academy of Business and Administrative Sciences Conference. Quebec City, Canada. 2001.

23. Burt J., Garman M. Conditional Monte Carlo: A simulation technique for stochastic network analysis. Management Science. 1971. No. 3(18). Pp. 207-217.

24. Van Slyke R. Monte Carlo methods and the PERT problem. Operations Research. 1963. Vol. 13. Pp. 141143.

25. Kalugin Yu.B. Universal'nyi metod otsenki srokov vypolneniya proekta $s$ veroyatnostnymi vremennymi parametrami [Universal method of computing finish time for networks with imprecise durations]. News of Higher Educational Institutions. Construction. 2015. No. 1. Pp. 51-59. (rus)

26. Tekhnologicheskie karty na ustroistvo zemlyanogo polotna i dorozhnoi odezhdy [Flow sheets on a construction of a road bed and a paving]. Moscow: ROSAVTODOR. 2004. 462 p. (rus)

Yuri Kalugin,

+7(905)2162825; yuri_kalugin@inbox.ru uncertainty: Survey and research potentials // European Journal of Operational Research. 2005. № 165. Pp. 289306.

19. Liberatore M. Critical path analysis with fuzzy activity times // Journal of Transactions on Engineering Management. 2008. Vol. 55. № 2. Pp. 329-337.

20. Klingel A. Bias in PERT completion times calculations for a real network // Management Science. 1966. Vol. 13. № 4. Pp. 476-489.

21. Schonberger R. Why projects are always late: a rationale based on manual simulation of a PERT/CPM network // Interfaces. 1981. Vol. 11. № 5. Pp. 66-70.

22. Haga W., O'Keefe T. Crashing PERT networks: A simulation approach // Proc. of the 4th International conference of the Academy of Business and Administrative Sciences Conference. Quebec City, Canada. 2001.

23. Burt J., Garman M. Conditional Monte Carlo: A simulation technique for stochastic network analysis // Management Science. 1971. № 3(18). Pp. 207-217.

24. Van Slyke R. Monte Carlo methods and the PERT problem // Operations Research. 1963. Vol. 13. Pp. 141-143.

25. Калугин Ю.Б. Универсальный метод оценки сроков выполнения проекта с вероятностными временными параметрами// Известия вузов. Строительство. 2015. № 1. С. 51-59.

26. Технологические карты на устройство земляного полотна и дорожной одежды. М.: РОСАВТОДОР. 2004. $462 \mathrm{c}$.

Юрий Борисович Калугин, $+7(905) 2162825$; эл. почma: yuri_kalugin@inbox.ru

(C) Kalugin Yu.B., 2017

Kalugin Yu.B. Universal method for calculation of reliable completion times. Magazine of Civil Engineering. 2016. No. 7. Pp. 70-80. doi: 10.5862/MCE.67.7 